\title{
Motion of a Localised Klein-Gordon Field
}

\author{
L. KOCIS* \\ Julius Kruttschnitt Mineral Research Centre, The University of Queensland \\ Isles Road, Indooroopilly, Queensland 4068, Australia
}

(Received September 19, 2001)

\begin{abstract}
Definitions of the momentum density, energy density, and densities of some other physical quantities are added to the standard Klein-Gordon theory. The densities introduced allow calculation of momentum and energy and their uncertainties of a localised Klein-Gordon field. It is shown that at certain conditions the momentum, energy, and potential energy of a localised Klein-Gordon field satisfy the relativistic Hamilton-Jacobi equation.
\end{abstract}

PACS numbers: 03.65.-W

\section{Introduction}

The classical limit of non-relativistic quantum mechanics can be demonstrated by a number of different approaches. The most common way from non-relativistic quantum mechanics to classical mechanics is to take the Ehrenfest theorem

$$
\frac{\mathrm{d}^{2}}{\mathrm{~d} t^{2}}\langle r\rangle=\frac{1}{m_{0}}\langle-\nabla U(r)\rangle
$$

and turn it into the Newton law of force for the centroid of a small wave packet

$$
\frac{\mathrm{d}^{2}}{\mathrm{~d} t^{2}}\langle\boldsymbol{r}\rangle=-\frac{1}{m_{0}} \nabla\langle\boldsymbol{r}\rangle(\langle\boldsymbol{r}\rangle)
$$

see for instance Ref. [1], p. 50. Another common option is to use an appropriate exponential substitution for the wave function such as

${ }^{*}$ Current address: Dr. L. Kocis, ALENEX, Peranga Court, Unit 4, 43 Fifth Avenue, Sandgate, QLD 4017, Australia. 


$$
\psi=\exp \left(\mathrm{i} \frac{S}{\hbar}\right)
$$

When the substitution (1.3) is applied in the Schrödinger equation

$$
\mathrm{i} \hbar \frac{\partial}{\partial t} \psi=\frac{1}{2 m_{0}}(-\mathrm{i} \hbar \nabla)^{2} \psi+U \psi
$$

it yields the relation

$$
-\frac{\partial S}{\partial t}=\frac{1}{2 m_{0}}(\nabla S)^{2}+U-\frac{\mathrm{i} \hbar}{2 m_{0}} \nabla^{2} S
$$

see for instance Ref. [1], p. 69. If the wave function has the form of a wave packet that is different from zero only in a small region around the centre of wave packet, $\langle r\rangle$, then the mean value of the gradient of the potential function can be expanded as

$$
\left\langle-\frac{\partial U}{\partial x_{i}}\right\rangle=-\frac{\partial U\left(\left\langle x_{i}\right\rangle\right)}{\partial\left\langle x_{i}\right\rangle}-\frac{1}{2} \frac{\partial^{3} U\left(\left\langle x_{i}\right\rangle\right)}{\partial\left\langle x_{i}\right\rangle^{3}}\left\langle\left(\Delta x_{i}\right)^{2}\right\rangle+\ldots, \quad i=1,2,3,
$$

see Ref. [1], p. 50.

The purpose of the classical limit of quantum mechanics is to show that at certain appropriate conditions such as

$$
\left\langle(\Delta r)^{2}\right\rangle \approx 0
$$

and

$$
\left\langle(\Delta p)^{2}\right\rangle \approx 0
$$

or

$$
(\nabla S)^{2} \gg \hbar\left|\nabla^{2} S\right|
$$

the quantum equations can be approximated and thus become classical equations. Indeed, Eq. (1.2) can be obtained from Eq. (1.1) assuming a smooth potential and then using expansion (1.6) and condition (1.7). However, for the right transition to classical mechanics conditions (1.7) and (1.8) have to be applied together. The simultaneous validity of (1.7) and (1.8) is possible for the motion of a particle with a large momentum in a smoothly varying external field. The classical Hamilton-Jacobi equation can be obtained from Eq. (1.5) using condition (1.9).

Because of the simplicity of the mathematical apparatus of non-relativistic quantum mechanics, its classical limit was obtained at the time when quantum mechanics was born [2-7]. Nevertheless, certain aspects and details of the classical limit of non-relativistic quantum mechanics are discussed until this day [8-17].

The two important equations of relativistic quantum mechanics are the Dirac equation (e.g. Ref. [1], p. 218) describing half-spin particles, and the Klein-Gordon equation $[18,19]$ describing zero-spin particles. Breen and Skiff [20] and 
Hestenes [21] have shown that the Dirac equation

$$
\mathrm{i} \hbar \frac{\partial \psi}{\partial t}=\left[c \alpha \cdot\left(-\mathrm{i} \hbar \nabla-\frac{e}{c} \boldsymbol{A}\right)+m_{0} c^{2} \beta+e \phi\right] \psi
$$

can be manipulated to give the relativistic Hamilton-Jacobi equation

$$
\left(\frac{\partial \theta}{\partial t}+e \phi\right)^{2}=c^{2}\left(\nabla \theta-\frac{e}{c} \boldsymbol{A}\right)^{2}+m_{0}^{2} c^{4}
$$

while no conditions like (1.7), (1.8), (1.9) or

$$
\hbar \rightarrow 0
$$

are required. (Equation (1.11) is obtained from Eq. (1.10) using substitution

$$
\psi=R \exp \left(\frac{\mathrm{i}}{\hbar} \theta\right)
$$

where $R$ is a complex four component column matrix, and $\theta$ is a real function.)

The derivation of the relativistic Hamilton-Jacobi equation from the Dirac equation implies the existence of the classical (understand non-quantum relativistic) solutions to the Dirac equation (1.10). This situation is quite different from that in the non-relativistic quantum mechanics where the equations of classical mechanics are obtained only if appropriate conditions, such as (1.7), (1.8), (1.9) or (1.12), are used.

Breen and Skiff have also shown that the Klein-Gordon equation for the stationary state of a charged particle

$$
(E-e \phi)^{2} \psi=c^{2}(-\mathrm{i} \hbar \nabla)^{2} \psi+m_{0}^{2} c^{4} \psi
$$

and substitution (1.13) where $R$ and $\theta$ are real functions give equation

$$
(E-e \phi)^{2} \psi=c^{2}(\nabla \theta)^{2} \psi+m_{0}^{2} c^{4} \psi-\hbar^{2} c^{2} \nabla^{2} R / R .
$$

According to Breen and Skiff [20], p. 591, Eq. (1.15) turns into the relativistic Hamilton-Jacobi equation for the stationary states if the quantum term there is set to zero

$$
\hbar^{2} c^{2} \nabla^{2} R / R=0
$$

or, the potential $\phi$ includes a term contributing a constant to Eq. (1.15) of the form

$$
V_{q}=-\hbar^{2} c^{2} \nabla^{2} R / R=\text { const. }
$$

Equations (1.16) and (1.17) give a class of potentials for which the relativistic Hamilton-Jacobi equation for the stationary states is exactly obtained. However, what happens for all the other potentials that are not specified with conditions (1.16) and (1.17)? The Schrödinger equation (1.4) gives the classical equations at certain conditions, such as (1.7) and (1.8), or (1.9). Equation (1.4) gives the classical Hamilton-Jacobi equation precisely if the quantum term in Eq. (1.5) is set 
to zero. (This would be similar to the approach of Breen and Skiff, see the conditions (1.16) and (1.17).) The Dirac equation gives the relativistic Hamilton-Jacobi equation without any conditions like (1.7)-(1.9). What about the Klein-Gordon equation? Does the Klein-Gordon equation give the relativistic Hamilton-Jacobi equation? If yes, then at what conditions?

The concern of this paper is to find out what happens when the Klein-Gordon equation is applied to the description of a charged particle in an external electromagnetic field at conditions when the quantum (or matter wave) phenomena are insignificant, and the external potential is arbitrary. It is shown that if we assume certain conditions, the Klein-Gordon equation yields the relativistic Hamilton-Jacobi equation. In this way it is demonstrated that the Klein-Gordon equation has the same property as the Schrödinger and the Dirac equations, namely to describe both quantum and non-quantum phenomena while no restrictions on external potentials need to be imposed.

\section{Properties of the Klein-Gordon equation}

The Klein-Gordon equation for a free particle is obtained from the relativistic relation between the kinetic energy and momentum

$$
\frac{E^{2}}{c^{2}}=p^{2}+m_{0}^{2} c^{2}
$$

in a manner that the physical quantities $E$ and $p$ in (2.1) are replaced by their operators

$$
\hat{E}=\mathrm{i} \hbar \frac{\partial}{\partial t}
$$

and

$$
\hat{\boldsymbol{p}}=-\mathrm{i} \hbar \nabla
$$

that act on a function $\psi$

$$
-\frac{\hbar^{2}}{c^{2}} \frac{\partial^{2}}{\partial t^{2}} \psi=-\hbar^{2} \Delta \psi+m_{0}^{2} c^{2} \psi
$$

Considering a charged particle in an external electromagnetic field, Eq. (2.4) is generalised to

$$
\left(\mathrm{i} \hbar \frac{\partial}{\partial t}-e \phi\right)^{2} \psi=c^{2}(-\mathrm{i} \hbar \nabla-e \boldsymbol{A})^{2} \psi+m_{0}^{2} c^{4} \psi
$$

e.g. Ref. [1], p. 209.

If the Klein-Gordon equation (2.5) is multiplied from left by $\psi^{*}$ and subtracted with the conjugate of (2.5) that was multiplied from the left by $\psi$, it gives the continuity equation 


$$
\frac{\partial \rho}{\partial t}+\nabla \cdot j=0
$$

where density $\rho$ and density current $j$ are defined with formulae

$$
\rho=\frac{\mathrm{i} \hbar}{2 m_{0} c^{2}}\left(\psi^{*} \frac{\partial \psi}{\partial t}-\psi \frac{\partial \psi^{*}}{\partial t}\right)-\frac{e \phi}{m_{0} c^{2}} \psi^{*} \psi
$$

and

$$
j=\frac{\hbar}{2 \mathrm{i} m_{0}}\left(\psi^{*} \nabla \psi-\psi \nabla \psi^{*}\right)-\frac{e \boldsymbol{A}}{m_{0}} \psi^{*} \psi .
$$

Density $\rho$ defined by (2.7) is not definite positive. For this reason it is impossible to interpret $\rho$ as the particle probability density, Ref. [1], p. 194. Pauli and Weisskopf [22] suggested the quantities (2.7) and (2.8) be considered as the electric charge density and the charge density current. With this assumption the number of the particles does not need to be conserved, only the electric charge is conserved. Therefore, the Klein-Gordon equation can be taken as a fundamental equation of a one-charge theory.

To obtain the charge density and the charge density current with proper physical dimensions, each of the two definitions above is multiplied by $e$. In this way we get

$$
\rho_{e}=\frac{\mathrm{i} \hbar e}{2 m_{0} c^{2}}\left(\psi^{*} \frac{\partial \psi}{\partial t}-\psi \frac{\partial \psi^{*}}{\partial t}\right)-\frac{e^{2} \phi}{m_{0} c^{2}} \psi^{*} \psi
$$

and

$$
j_{e}=\frac{\hbar e}{2 \mathrm{i} m_{0}}\left(\psi^{*} \nabla \psi-\psi \nabla \psi^{*}\right)-\frac{e^{2} \boldsymbol{A}}{m_{0}} \psi^{*} \psi .
$$

The fact that the number of the particles is not conserved while the charge is, can be interpreted by annihilation and creation of pairs of particles with opposite signs. This interpretation, however, can be consistently implemented only using field theories. If there is only one particle, the charge density $\rho_{e}$ is either positive or negative in dependence on the sign of the charge of the particle, Ref. [1], p. 195. For the neutral particles $\rho_{e}=0$. In this paper we will consider only charged particles.

Substitution (1.13) where $R$ and $\theta$ are real functions applied in formula for the charge density (2.9) turns the latter into equation

$$
\rho_{e}=\frac{e}{m_{0} c^{2}}\left(-R^{2} \frac{\partial \theta}{\partial t}-R^{2} e \phi\right) .
$$

Further we will consider only cases satisfying condition

$$
-\frac{\partial \theta}{\partial t}-e \phi>0
$$

so the charge density (2.9) becomes definite positive. (We suppose that the elementary electric charge $e$ is positive. In the case of a negatively charged particle the charge is $-e$ and the charge density given by (2.9) and (2.11) becomes definite 
negative.) The charge density that is definite positive (or definite negative) allows that the one-charge theory becomes also a one-particle theory. At these conditions the charge density can be normalised as

$$
e=\int \rho_{e} \mathrm{~d}^{3} r
$$

and

$$
1=\int \rho \mathrm{d}^{3} r
$$

In further text we will consider a single particle with charge $e$ in an external electromagnetic field at condition (2.12). Additionally, we will assume that the particle is described with a localised Klein-Gordon field. Such field is formed by superposition of relativistic waves with momenta from a narrow band, and is a relativistic analogue of non-relativistic wave packets. The localised Klein-Gordon field, or a relativistic wave packet, moves with a velocity, can be ascribed physical quantities such as energy and momentum, and it has to obey certain rules.

The intention of this paper is to find these rules, or the equations of motion of a localised Klein-Gordon field for a stationary external electromagnetic field specified with conditions

$$
\boldsymbol{A}=0
$$

and

$$
\phi(r, t) \equiv \phi(r)
$$

To achieve this goal we will introduce the density of momentum, the density of energy, and densities of some other physical quantities. Integration of the density of momentum over the localised Klein-Gordon field gives the momentum of that field. The same can be done for densities of other physical quantities. Expressions for the physical quantities of a localised Klein-Gordon field are derived in Secs. 3-7. These include the velocity, charge current, momentum, momentum squared, uncertainty of momentum, energy, energy squared, and uncertainty of energy. Having obtained the expressions for the quantities listed, the equation of motion of the localised Klein-Gordon field is found in Sec. 8.

\section{Velocity of the localised Klein-Gordon field}

A localised Klein-Gordon field and a small variation in $\partial \theta / \partial t$ and $\phi$ within that field facilitate approximations

$$
\int R^{2} \frac{\partial \theta}{\partial t} \mathrm{~d}^{3} r=\frac{\partial \theta(\langle r\rangle)}{\partial t} \int R^{2} \mathrm{~d}^{3} r
$$

and 


$$
\int R^{2} \phi \mathrm{d}^{3} r=\phi(\langle r\rangle) \int R^{2} \mathrm{~d}^{3} r
$$

Approximations (3.1) and (3.2) are obtained from the expansions of functions $\partial \theta / \partial t$ and $\phi$ in a similar way as the approximation

$$
\langle\nabla U(\boldsymbol{r})\rangle=\nabla_{\langle\pi\rangle} U(\langle\boldsymbol{r}\rangle)
$$

is obtained from the expansion (1.6). Approximations (3.1) and (3.2) allow that applying (2.11) in (2.13) gives

$$
m_{0} c^{2}=\left(-\frac{\partial \theta(\langle r\rangle)}{\partial t}-e \phi(\langle r\rangle)\right) \int R^{2} \mathrm{~d}^{3} r
$$

Substitution (1.13) in the charge density current (2.10) leads to equation

$$
j_{e}=\frac{e}{m_{0}} R^{2}(\nabla \theta-e \boldsymbol{A}) .
$$

Assuming a localised Klein-Gordon field, and a small variation of $\nabla \theta$ within that field allow approximation

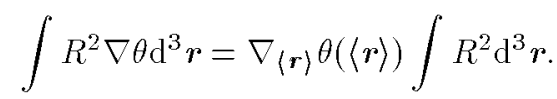

Assuming a stationary external field, see conditions (2.15) and (2.16), and using approximation (3.5) the integral of $j_{e}$ is

$$
\int j_{e} \mathrm{~d}^{3} r=\frac{e}{m_{0}} \nabla_{\langle\boldsymbol{r}\rangle} \theta(\langle r\rangle) \int R^{2} \mathrm{~d}^{3} r
$$

Let us consider now the formula for the current created by the translational movement of a volume element of charge density $\rho_{e}$ and velocity $v$ :

$$
j_{e}=\rho_{e} v
$$

Formula (3.7) can be also applied to the whole body of the localised Klein-Gordon field, which we write as

$$
\int j_{e} \mathrm{~d}^{3} \boldsymbol{r}=\langle\boldsymbol{v}\rangle \int \rho_{e} \mathrm{~d}^{3} \boldsymbol{r},
$$

where

$$
\langle v\rangle=\frac{\mathrm{d}}{\mathrm{d} t}\langle r\rangle
$$

is the velocity and

$$
\langle r\rangle=\int \rho r \mathrm{~d}^{3} r
$$

is the centroid of the localised Klein-Gordon field. Using (3.6) and (2.13) in (3.8) gives

$$
\nabla_{\langle\boldsymbol{r}\rangle} \theta(\langle r\rangle) \int R^{2} \mathrm{~d}^{3} r=m_{0}\langle\boldsymbol{v}\rangle
$$




\section{Density of momentum and momentum}

The charge density $\rho_{e}$ is defined as

$$
\rho_{e}=\rho e,
$$

compare with Eqs. (2.7) and (2.9). Analogically to the definition of the charge density (4.1) we define the momentum density of a localised Klein-Gordon field as

$$
\rho_{\mathbb{P}}=\rho \psi^{-1} \hat{p} \psi
$$

where the momentum operator is given by (2.3). Substitution (1.13) and Eqs. (2.11) and (4.1) applied in (4.2) change the right side of (4.2)

$$
\rho_{\mathbb{P}}=\frac{R^{2}}{m_{0} c^{2}}\left(-\frac{\partial \theta}{\partial t}-e \phi\right)\left(-\mathrm{i} \hbar \frac{\nabla R}{R}+\nabla \theta\right) .
$$

Since the momentum density is defined with (4.2), the momentum of the localised Klein-Gordon field has to be defined with integral

$$
\langle p\rangle=\int \rho_{\boldsymbol{p}} \mathrm{d}^{3} r
$$

The mean value of the momentum of the localised Klein-Gordon field is also defined with Eq. (4.4). The expression for the momentum density given by (4.3) applied in Eq. (4.4) splits the right side of Eq. (4.4) into two integrals

$\langle p\rangle=-\frac{\mathrm{i} \hbar}{m_{0} c^{2}} \int\left(-\frac{\partial \theta}{\partial t}-e \phi\right) R \nabla R \mathrm{~d}^{3} r+\frac{1}{m_{0} c^{2}} \int\left(-\frac{\partial \theta}{\partial t}-e \phi\right) R^{2} \nabla \theta \mathrm{d}^{3} r$.

Thank to approximations (3.1) and (3.2) the term in the brackets of the first integral can be moved in front of the integral sign. The first integral in (4.5) is then zero. This happens because function $R \nabla R$ can be written as $\nabla\left(R^{2}\right) / 2$ and the integral of function $\nabla\left(R^{2}\right) / 2$ is zero. In the second integral above we could move the expression in the brackets in front of the integral sign as well, but we do it only with function $\nabla \theta$ :

$$
\langle p\rangle=\nabla_{\langle\tau\rangle} \theta(\langle r\rangle) \frac{1}{m_{0} c^{2}} \int\left(-\frac{\partial \theta}{\partial t}-\epsilon \phi\right) R^{2} \mathrm{~d}^{3} r .
$$

From (2.11) and (2.13) it follows that the value of integral in (4.6) including the constant in front of it is 1 . Hence

$$
\langle p\rangle=\nabla_{\langle r\rangle} \theta(\langle r\rangle) .
$$

Equations (4.7) and (3.10) give

$$
\langle p\rangle=m_{0}\langle v\rangle\left(\int R^{2} \mathrm{~d}^{3} r\right)^{-1}
$$

Since the value of the integral in Eq. (4.8) is contained in Eq. (3.3), the right side of Eq. (4.8) can be rewritten in terms of $\langle v\rangle, \theta$, and $\phi$ : 


$$
\langle p\rangle=m_{0}\langle v\rangle \frac{1}{m_{0} c^{2}}\left(-\frac{\partial \theta(\langle\boldsymbol{r}\rangle)}{\partial t}-e \phi(\langle\boldsymbol{r}\rangle)\right) .
$$

It is tempting to express the function in the brackets on the right side of (4.9) in terms of the velocity of the localised Klein-Gordon field $\langle v\rangle$. This will be done in Sec. 8 .

\section{Density of momentum squared, momentum squared and dispersion of momentum}

Density of momentum squared of a localised Klein-Gordon field is defined with formula

$$
\rho_{p^{2}}=\rho \psi^{-1}(\hat{\boldsymbol{p}})^{2} \psi
$$

Substitution (1.13) together with Eqs. (2.11) and (4.1) expand the right side of (5.1) into three terms

$$
\begin{aligned}
\rho_{\mathbb{P}^{2}} & =\frac{-\hbar^{-2}}{m_{0} c^{2}}\left(-\frac{\partial \theta}{\partial t}-\epsilon \phi\right) R \Delta R-\frac{\mathrm{i} \hbar}{m_{0} c^{2}}\left(-\frac{\partial \theta}{\partial t}-\epsilon \phi\right) \nabla\left(R^{2} \nabla \theta\right) \\
& +\frac{R^{2}}{m_{0} c^{2}}\left(-\frac{\partial \theta}{\partial t}-e \phi\right)(\nabla \theta)^{2} .
\end{aligned}
$$

The momentum squared of a localised Klein-Gordon field is defined with integral

$$
\left\langle p^{2}\right\rangle=\int \rho_{\mathbb{P}^{2}} \mathrm{~d}^{3} r
$$

The mean value of the momentum squared of a localised Klein-Gordon field is also given with (5.3). To determine the integral (5.3) we have to evaluate the integrals of the three terms on the right side of (5.2). The integral of the first term on the right side of Eq. (5.2) cannot be manipulated, so it will be left unchanged. Considering the integral of the second term on the right side of Eq. (5.2), expression $(\partial \theta / \partial t-e \phi)$ can be moved in front of the integral sign because the appreciable values of $R^{2}$ occur only around $\langle r\rangle$ and functions $\partial \theta / \partial t, \nabla \theta$, and $\phi$ do not vary significantly within the body of the wave packet. The integral of the rest, namely of the function $\nabla\left(R^{2} \nabla \theta\right)$ is zero, so the integral of the whole second term on the right side of (5.2) is zero. Taking the integral of the third term on the right side of (5.2), function $(\nabla \theta)^{2}$ can be moved in front of the integral sign. The integral of the remaining part of the third term in (5.2) is then 1. This follows from Eqs. (2.11), (2.13) and (2.14). Hence

$$
\left\langle p^{2}\right\rangle=\frac{-\hbar^{-2}}{m_{0} c^{2}} \int R \Delta R\left(-\frac{\partial \theta}{\partial t}-e \phi\right) \mathrm{d}^{3} r+[\nabla \theta(\langle r\rangle)]^{2} .
$$

The dispersion of momentum of a localised Klein-Gordon field is defined with formula

$$
\left\langle(\Delta p)^{2}\right\rangle=-\langle p\rangle^{2}+\left\langle p^{2}\right\rangle .
$$


The first term on the right side of Eq. (5.5) can be substituted with (4.7) and the second term on the right side of (5.5) equals to (5.4). Hence the dispersion of momentum of a localised Klein-Gordon field is

$$
\left\langle(\Delta p)^{2}\right\rangle=\frac{-\hbar^{-2}}{m_{0} c^{2}} \int R \Delta R\left(-\frac{\partial \theta}{\partial t}-e \phi\right) \mathrm{d}^{3} r .
$$

\section{Density of energy and energy}

For stationary external fields (see conditions (2.15) and (2.16)) the energy density of a localised Klein-Gordon field is defined with formula

$$
\rho_{E}=\rho \psi^{-1} \hat{E} \psi
$$

Expression (2.11), substitution (1.13) and operator (2.2) allow to express the right side of Eq. (6.1)

$$
\rho_{E}=\frac{1}{m_{0} c^{2}}\left(-\frac{\partial \theta}{\partial t}-e \phi\right)\left(\mathrm{i} \hbar R \frac{\partial R}{\partial t}-R^{2} \frac{\partial \theta}{\partial t}\right) .
$$

The energy of a localised Klein-Gordon field is the integral of (6.1),

$$
\langle E\rangle=\int \rho_{E} \mathrm{~d}^{3} r
$$

so we get

$$
\begin{aligned}
\langle E\rangle & =\frac{\mathrm{i} \hbar}{m_{0} c^{2}} \int\left(-\frac{\partial \theta}{\partial t}-\epsilon \phi\right) R \frac{\partial R}{\partial t} \mathrm{~d}^{3} \boldsymbol{r} \\
& +\frac{1}{m_{0} c^{2}} \int\left(-\frac{\partial \theta}{\partial t}-e \phi\right)\left(-R^{2} \frac{\partial \theta}{\partial t}\right) \mathrm{d}^{3} r .
\end{aligned}
$$

Equations (6.3) and (6.4) also define the mean energy of a localised Klein-Gordon field. In order to evaluate the first integral in (6.4) we rewrite Eq. (2.14) as

$$
1=\frac{1}{m_{0} c^{2}} \int R^{2}\left(-\frac{\partial \theta}{\partial t}-\epsilon \phi\right) \mathrm{d}^{3} r
$$

The partial time derivative of Eq. (6.5) gives

$$
2 \int\left(-\frac{\partial \theta}{\partial t}-e \phi\right) R \frac{\partial R}{\partial t} \mathrm{~d}^{3} r=-\int\left(-\frac{\partial^{2} \theta}{\partial t^{2}}-e \frac{\partial \phi}{\partial t}\right) R^{2} \mathrm{~d}^{3} r
$$

The partial time derivative of the potential function on the right side of $(6.6)$ is zero because of condition (2.16). The second partial time derivative of function $\theta$ can be neglected because the first partial time derivative of $\theta$ does not vary appreciably within the localised Klein-Gordon field. Later it will be shown that the second partial time derivative of function $\theta$ is zero because for static fields (see conditions (2.15) and (2.16)) the first partial time derivative of $\theta$ is a constant. 
Now if the right side of (6.6) is zero, then the left side of (6.6) is zero, and so is the first integral on the right side of (6.4). Function $\partial \theta / \partial t$ in the second brackets of the second integral on the right side of (6.4) can be moved in front of the integral sign, so we get

$$
\langle E\rangle=-\frac{\partial \theta(\langle r\rangle)}{\partial t} \frac{1}{m_{0} c^{2}} \int\left(-\frac{\partial \theta}{\partial t}-e \phi\right) R^{2} \mathrm{~d}^{3} r
$$

Equations (2.11), (2.13), and (2.14) imply that the value of the integral on the right side of (6.7) is 1 . Therefore Eq. (6.7) simplifies to

$$
\langle E\rangle=-\frac{\partial \theta(\langle\boldsymbol{r}\rangle)}{\partial t}
$$

\section{Density of energy squared, energy squared, and dispersion of energy} formula

Density of energy squared of a localised Klein-Gordon field is defined with

$$
\rho_{E^{2}}=\rho \psi^{-1}(\hat{E})^{2} \psi
$$

Substitution (1.13) and the formula for density (2.11) applied in Eq. (7.1) give

$$
\begin{aligned}
\rho_{E^{2}} & =\frac{1}{m_{0} c^{2}}\left(-\frac{\partial \theta}{\partial t}-e \phi\right) \\
& \times\left[-\hbar^{2} R \frac{\partial^{2} R}{\partial t^{2}}-2 \mathrm{i} \hbar R \frac{\partial R}{\partial t} \frac{\partial \theta}{\partial t}-\mathrm{i} \hbar R^{2} \frac{\partial^{2} \theta}{\partial t^{2}}+R^{2}\left(\frac{\partial \theta}{\partial t}\right)^{2}\right] .
\end{aligned}
$$

The integral of (7.2) is the energy squared and the average of the energy squared of the Klein-Gordon field

$$
\begin{aligned}
\left\langle E^{2}\right\rangle & =\int \rho_{E^{2}} \mathrm{~d}^{3} r=\frac{-\hbar^{2}}{m_{0} c^{2}} \int\left(-\frac{\partial \theta}{\partial t}-e \phi\right) R \frac{\partial^{2} R}{\partial t^{2}} \mathrm{~d}^{3} r \\
& -\frac{2 \mathrm{i} \hbar}{m_{0} c^{2}} \int\left(-\frac{\partial \theta}{\partial t}-e \phi\right) R \frac{\partial R}{\partial t} \frac{\partial \theta}{\partial t} \mathrm{~d}^{3} r-\frac{\mathrm{i} \hbar}{m_{0} c^{2}} \int\left(-\frac{\partial \theta}{\partial t}-e \phi\right) R^{2} \frac{\partial^{2} \theta}{\partial t^{2}} \mathrm{~d}^{3} r \\
& +\frac{1}{m_{0} c^{2}} \int\left(-\frac{\partial \theta}{\partial t}-e \phi\right) R^{2}\left(\frac{\partial \theta}{\partial t}\right)^{2} \mathrm{~d}^{3} r
\end{aligned}
$$

The first integral on the right side of (7.3) will be left without manipulations. The derivative $\partial \theta / \partial t$ in the second integral on the right side of (7.3) that is not in brackets can be moved in front of the integral sign. The second integral on the right side of (7.3) is then the same as that on the left side of Eq. (6.6), which is zero. Therefore the second integral on the right side of (7.3) is zero. The second 
partial time derivative of $\theta$ in the third integral on the right side of Eq. (7.3) is approximated as zero because $\partial \theta / \partial t$ does not change appreciably within the localised Klein-Gordon field. Also, as it was already mentioned in reference to Eq. (6.6), later it will be shown that the second partial time derivative of $\theta$ is zero. Thus the third integral on the right side of Eq. (7.3) is zero. In the fourth integral on the right side of Eq. (7.3) term $(\partial \theta / \partial t)^{2}$ can be extracted from the integral. The integral of the remaining terms is then 1, which follows from Eqs. (2.11), (2.13), and (2.14). In this way Eq. (7.3) simplifies to

$$
\left\langle E^{2}\right\rangle=\frac{-\hbar^{2}}{m_{0} c^{2}} \int\left(-\frac{\partial \theta}{\partial t}-\epsilon \phi\right) R \frac{\partial^{2} R}{\partial t^{2}} \mathrm{~d}^{3} r+\left(\frac{\partial \theta(\langle\boldsymbol{r}\rangle)}{\partial t}\right)^{2}
$$

The energy dispersion of a localised Klein-Gordon field is defined as

$$
\left\langle(\Delta E)^{2}\right\rangle=-\langle E\rangle^{2}+\left\langle E^{2}\right\rangle .
$$

Equations (6.8) and (7.4) applied in the right side of (7.5) give

$$
\left\langle\left(\Delta E^{2}\right)\right\rangle=\frac{-\hbar^{2}}{m_{0} c^{2}} \int\left(-\frac{\partial \theta}{\partial t}-e \phi\right) R \frac{\partial^{2} R}{\partial t^{2}} \mathrm{~d}^{3} r
$$

\section{Transition to the relativistic Hamilton-Jacobi equation for a stationary external field}

Substitution (1.13) and condition (2.15) applied in the Klein-Gordon equation (2.5) partition this equation into its real and imaginary parts that are

$$
-\hbar^{2} \frac{\partial^{2} R}{\partial t^{2}}+\left(\frac{\partial \theta}{\partial t}+e \phi\right)^{2} R=-c^{2} \hbar^{2} \Delta R+c^{2}(\nabla \theta)^{2} R+m_{0}^{2} c^{4} R
$$

and

$$
2 \frac{\partial R}{\partial t} \frac{\partial \theta}{\partial t}+R \frac{\partial^{2} \theta}{\partial t^{2}}+2 e \phi \frac{\partial R}{\partial t}=2 c^{2} \nabla \cdot(R \nabla \theta) .
$$

Equations (8.1) and (8.2) were firstly derived by Takabayasi [23]. They are also given by Breen and Skiff [20]. Equation (8.1) is the relativistic Hamilton-Jacobi equation with two additional quantum terms. These are the first term on the left side of (8.1) containing the second partial time derivative of $R$, and the first term on the right side of (8.1) containing the second partial spacial derivatives of $R$. Equation (8.2) is equivalent to the relativistic continuity equation (2.6). This can be demonstrated if the substitution (1.13) is applied in (2.7) and (2.8), and then these are used in Eq. (2.6).

Equation (8.1) is now multiplied from the left by

$$
\frac{1}{m_{0} c^{2}}\left(-\frac{\partial \theta}{\partial t}-e \phi\right) R
$$

and integrated over the whole space, or, just over the volume of the localised Klein-Gordon field, to give 


$$
\begin{aligned}
& \frac{-\hbar^{2}}{m_{0} c^{2}} \int\left(-\frac{\partial \theta}{\partial t}-e \phi\right) R \frac{\partial^{2} R}{\partial t^{2}} \mathrm{~d}^{3} r+\frac{1}{m_{0} c^{2}} \int\left(-\frac{\partial \theta}{\partial t}-e \phi\right) R^{2}\left(-\frac{\partial \theta}{\partial t}-e \phi\right)^{2} \mathrm{~d}^{3} r \\
& =c^{2} \frac{-\hbar^{2}}{m_{0} c^{2}} \int R \Delta R\left(-\frac{\partial \theta}{\partial t}-e \phi\right) \mathrm{d}^{3} r+c^{2} \frac{1}{m_{0} c^{2}} \int\left(-\frac{\partial \theta}{\partial t}-e \phi\right) R^{2}(\nabla \theta)^{2} \mathrm{~d}^{3} r \\
& +m_{0}^{2} c^{4} \frac{1}{m_{0} c^{2}} \int\left(-\frac{\partial \theta}{\partial t}-e \phi\right) R^{2} \mathrm{~d}^{3} r
\end{aligned}
$$

The first integral on the left side of Eq. (8.3) is the dispersion of energy, see Eq. (7.6), and the first integral on the right side of the same equation is the dispersion of momentum multiplied by the velocity of light squared, see Eq. (5.6). In the second integral on the left side of Eq. (8.3) expression $(-\partial \theta / \partial t-e \phi)^{2}$ is approximated as $[-\partial \theta(\langle\boldsymbol{r}\rangle) / \partial t-e \phi(\langle\boldsymbol{r}\rangle)]^{2}$ and moved in front of the integral. In the second integral on the right side of Eq. (8.3) function $(\nabla \theta)^{2}$ can be approximated as $\left[\nabla \theta_{\langle r\rangle}(\langle r\rangle)\right]^{2}$, and moved in front of the integral sign. These steps are authorised by the properties of functions $\partial \theta / \partial t, \phi$, and $\nabla \theta$ which, as already stated previously, do not vary appreciably within the localised Klein-Gordon field. The approximations just mentioned and Eqs. (2.11), (2.13), and (2.14) allow that the second integral on the left side of Eq. (8.3) is $[-\partial \theta(\langle\boldsymbol{r}\rangle) / \partial t-e \phi(\langle r\rangle)]^{2}$ and the second integral on the right side of Eq. (8.3) becomes $c^{2}\left[\nabla \theta_{\langle r\rangle}(\langle r\rangle)\right]^{2}$. Use of $(2.11)$, (2.13), and (2.14) in the third integral on the right side of Eq. (8.3) gives constant $m_{0}^{2} c^{4}$. Hence Eq. (8.3) can be rewritten as

$$
\left\langle(\Delta E)^{2}\right\rangle+\left(-\frac{\partial \theta(\langle r\rangle)}{\partial t}-e \phi(\langle r\rangle)\right)^{2}=c^{2}\left\langle(\Delta p)^{2}\right\rangle+c^{2}\left[\nabla \theta_{\langle r\rangle}(\langle r\rangle)\right]^{2}+m_{0}^{2} c^{4}
$$

Equation (8.4) is the relativistic Hamilton-Jacobi equation written for the centroid of a localised Klein-Gordon field with two additional "error" terms, namely the dispersion of energy and the dispersion of the momentum multiplied by the velocity of light squared. Note that the two "error" terms in Eq. (8.4) are always positive and thus they tend to cancel each other. Therefore when they do so, or, when they are small in comparison with the other terms in Eq. (8.4), Eq. (8.4) reduces to

$$
\left(-\frac{\partial \theta(\langle\boldsymbol{r}\rangle)}{\partial t}-e \phi(\langle\boldsymbol{r}\rangle)\right)^{2}=c^{2}\left[\nabla_{\langle\boldsymbol{r}\rangle} \theta(\langle\boldsymbol{r}\rangle)\right]^{2}+m_{0}^{2} c^{4}
$$

which is exactly the relativistic Hamilton-Jacobi equation written for the centroid of a localised Klein-Gordon field.

The physical meaning of function $\theta$ in Eqs. (8.4) and (8.5) is confirmed with Eqs. (4.7) and (6.8). If the external fields satisfy conditions (2.15) and (2.16) energy is conserved. Therefore $\langle E\rangle$ is a constant and according to Eq. (6.8) the derivative $\partial \theta / \partial t$ is also constant. It follows that the second time partial derivative of $\theta$ is zero and the integral on the right side of Eq. (6.6) is zero as well. 
Equation (4.9) attempting to relate the mean value of the momentum $\langle p\rangle$ and velocity $\langle v\rangle$ can be improved using (8.5). To do it, Eq. (4.9) is squared

$$
\langle p\rangle^{2}=m_{0}^{2}\langle v\rangle^{2} \frac{1}{m_{0}^{2} c^{4}}\left(-\frac{\partial \theta(\langle r\rangle)}{\partial t}-e \phi(\langle\boldsymbol{r}\rangle)\right)^{2} .
$$

Applying (4.7) in (8.5) and then applying the latter in (8.6) gives

$$
\langle p\rangle^{2}=m_{0}^{2}\langle v\rangle^{2} \frac{1}{m_{0}^{2} c^{4}}\left(c^{2}\langle p\rangle^{2}+m_{0}^{2} c^{4}\right) .
$$

Equation (8.7) can be solved for $\langle\boldsymbol{p}\rangle$ to give

$$
\langle p\rangle= \pm \frac{m_{0}\langle v\rangle}{\sqrt{1-\langle v\rangle^{2} / c^{2}}} .
$$

The negative sign in (8.8) has no physical interpretation. The momentum vector and the velocity vector have to have the same direction. Therefore we choose

$$
\langle p\rangle=\frac{m_{0}\langle v\rangle}{\sqrt{1-\langle v\rangle^{2} / c^{2}}} .
$$

Obviously, Eq. (8.9) relating the momentum and the velocity of the centroid of a localised Klein-Gordon field is subject to a small, or equal values of the dispersion of energy and the dispersion of momentum, see Eqs. (8.4) and (8.5). In the case when the dispersion of energy and the dispersion of momentum are not negligible, or they do not cancel each other, relation (8.9) has to be replaced by its more general form, namely

$$
\langle p\rangle=\frac{\sqrt{m_{0}^{2}+c^{-4}\left\langle(\Delta E)^{2}\right\rangle-c^{-2}\left\langle(\Delta p)^{2}\right\rangle}}{\sqrt{1-\langle v\rangle^{2} / c^{2}}}\langle v\rangle .
$$

Equation (8.10) can be obtained from Eqs. (4.7), (4.9), and (8.4).

\section{The classical limit of the Klein-Gordon equation}

The meaning of the classical limit of the Klein-Gordon equation discussed here and throughout the paper is the application of the Klein-Gordon equation to cases where the quantum, or matter wave phenomena become insignificant. The classical limit of the Klein-Gordon equation discussed here has nothing to do with the classical limit of the Klein-Gordon equation demonstrated by Davydov, where the Klein-Gordon equation reduces to the Schrödinger equation, see Ref. [1], p. 193 .

The formalism of the Klein-Gordon equation allows a column matrix wave function with two components, see e.g. Ref. [1], p. 197 and Refs. [24, 25]. In this approach the electromagnetic interaction combines the positive and negative frequency parts of the Klein-Gordon scalar field, so we cannot deal with particles and antiparticles separately. Besides that, the normalisation of the wave function is to a fixed volume $V$ that is not in movement. Normalisation of the wave 
function to a moving wave packet with a narrow spectrum of energies would introduce inconsistency because the velocity of the wave packet does not correspond to the velocity derived from the relations between operators. Note that in the Klein-Gordon theory with the component wave function the eigenvalues of the velocity operator come out with wrong values. (See for instance Ref. [1], p. 206.) Now if there was a way to fix these problems, then the small wave packet in an external electromagnetic field will immediately split into a part containing the particle and a part containing the antiparticle because the electromagnetic forces acting on the particle and the antiparticle are of opposite directions. Therefore, the whole theory would be problematic again because its interaction mechanism requires the presence of both the particle and the antiparticle. Similar difficulties would occur in the approach developed by Horwitz, see Refs. [26, 27]. This indicates that the classical limit of the Klein-Gordon theory with the two-component wave function can be scarcely achieved. On the other hand, the Klein-Gordon equation for one-component wave function (2.5) at condition (2.12) is a one-charge and one-particle theory, which for a small packet, or a localised Klein-Gordon field and smooth external potential gives exactly the relativistic Hamilton-Jacobi equation.

The derivations presented in Secs. 3-8 are valid for any smooth potential and the class of energies satisfying inequality (2.12).

The results obtained can be taken as approximately valid also for potentials where inequality (2.12) is satisfied in most parts of the space, while the rest of the space is filled with potential barriers significantly larger than $\langle E\rangle$, the energy of the particle. These potential barriers are assumed to be sufficiently wide, so that the probability of quantum tunneling is diminishingly small. When a localised Klein-Gordon field approaches such a high and large potential wall, its forward tail will penetrate into the region where (2.12) is not satisfied. However, at the same time, the amplitude of the forward tail is significantly suppressed by the large potential of the barrier. Hence the contribution of the Klein-Gordon field from the region where $\langle E\rangle\langle e \phi$ to the integral in (2.13) is negligible.

The derivation of the relativistic Hamilton-Jacobi equation (8.5) can be compared to the transition from quantum mechanics to classical mechanics that is done by means of the Ehrenfest theorem (1.1). Equation (1.1) is a part of the formalism of non-relativistic quantum mechanics. It can be derived from the Schrödinger equation without neglecting anything. Considering a small non-relativistic wave packet that does not spread too rapidly, see conditions (1.7) and (1.8), the right side of (1.1) can be approximated as (1.6), so we get immediately the Newton law of force written for the centroid of the wave packet (1.2). The analogy between (1.2) and (8.5) is obvious. Both of them are equations of motion for the centroid of a small field representing a single particle.

According to Davydov (see Ref. [1]) the transition from the Schrödinger equation to classical mechanics occurs if the uncertainties of the coordinates and 
momentum go to zero. The transition from the Klein-Gordon equation to classical mechanics is similar. The small uncertainty of the coordinates implies approximations (3.1), (3.2), and a similar approximation for $\nabla \theta$, see the text between Eqs. (8.3) and (8.4). When the uncertainties of the coordinates and momentum go to zero, the Heisenberg uncertainty relation implies that the Planck constant goes to zero as well. This signifies the classical limit of the Klein-Gordon equation. In this limit the coordinates of the centre of a very small wave packet can be replaced by the particle coordinates and Eq. (8.5) becomes the classical (understand relativistic) Hamilton-Jacobi equation known from classical (understand "non-quantum") physics.

The formalism presented should be well applicable also to the Schrödinger equation, some representations of the Klein-Gordon equation, to certain transformations of the Dirac equations, and to equations similar to the Dirac and Klein-Gordon equations such as those suggested by Kemmer [28] and Durand [29]. The author is presently pursuing these problems.

\section{References}

[1] D.I. Davydov, Quantum Mechanics, Pergamon, Oxford 1965; for the expansion (1.6) see also D.I. Blokhintsev, Quantum Mechanics, D. Reidel Pub. Co., Dordrecht 1964, p. 102.

[2] E. Madelung, Z. Phys. 40, 322 (1926).

[3] L. Brillouin, J. Phys. Radium 7, 353 (1926).

[4] H.A. Kramers, Z. Phys. 39, 518 (1926).

[5] G. Wentzel, Z. Phys. 38, 828 (1926).

[6] P. Ehrenfest, Z. Phys. 44, 455 (1927).

[7] J.H. Van Vleck, Proc. Natl. Acad. Sci. 14, 518 (1928).

[8] P.A.M. Dirac, The Principles of Quantum Mechanics, Oxford University, Oxford 1958, p. 121.

[9] N. Rosen, Am. J. Phys. 32, 597 (1964).

[10] L.S. Brown, Am. J. Phys. 40, 371 (1972).

[11] D.B. Berkowitz, P.D. Skiff, Am. J. Phys. 40, 1625 (1972).

[12] K. Hepp, Commun. Math. Phys. 35, 265 (1974); see also K. Yajima, Commun. Math. Phys. 69, 101 (1979); G. Hagedorn, Commun. Math. Phys. 71, 77 (1980).

[13] D. Home, S. Sengupta, Am. J. Phys. 51, 265 (1983).

[14] L.E. Ballentine, Y.M. Yang, J.P. Zibin, Phys. Rev. A 50, 2854 (1994); L.E. Ballentine, J.P. Zibin, Phys. Rev. A 54, 3813 (1996); L.E. Ballentine, S.M. McRae, Phys. Rev. A 58, 1799 (1998).

[15] G.W. Ford, R.F. O'Connell, Phys. Lett. A 215, 245 (1996).

[16] V. Aldaya, M. Calixto, J. Guerrero, Commun. Math. Phys. 178, 399 (1996). 
[17] A.J. Makowski, S. Konkel, Phys. Rev. A 58, 4975 (1998); A. Mostafazadeh, Nucl. Phys. B 509, 529 (1998); A. Mostafazadeh, Phys. Rev. A 60, 5144 (1999); A.J. Makowski, S. Konkel, Phys. Rev. A 60, 5146 (1999).

[18] O. Klein, Z. Phys. 37, 895 (1926); see also E. Schrödinger, Ann. Phys. 81, 109 (1926); V. Fock, Z. Phys. 38, 242 (1926); V. Fock, Z. Phys. 39, 226 (1926).

[19] W. Gordon, Z. Phys. 40, 117 (1927).

[20] S. Breen, P.D. Skiff, Found. Phys. 7, 589 (1977).

[21] D. Hestenes, in: NATO and SERC Workshop on Clifford Algebras and Their Applications in Mathematical Physics, Canterbury (England) 1985, Eds. J.S.R. Chisholm, A.K. Common, Kluwer Academic Publ., Dordrecht 1986, p. 102.

[22] W. Pauli, V. Weisskopf, Helv. Phys. Acta 7, 709 (1934).

[23] T. Takabayasi, Prog. Theor. Phys. 9, 197 (1953).

[24] H. Feshbach, F. Villars, Rev. Mod. Phys. 30, 24 (1958).

[25] G. Marx, Nuovo Cimento A 60, 669 (1969).

[26] L.P. Horwitz, C. Piron, Helv. Phys. Acta 46, 316 (1973).

[27] L.P. Horwitz, Found. Phys. 14, 1027 (1984).

[28] N. Kemmer, Proc. R. Soc. A 173, 91 (1939).

[29] E. Durand, Phys. Rev. D 11, 3405 (1975). 